\title{
3D homologous multi-points warping application to sexual dimorphism in human face
}

\begin{abstract}
Sexual dimorphism in Homo-sapiens is a phenomenon of a direct product of evolution by natural selection where evolutionary forces acted separately on the sexes which brought about the differences in appearance between male and female such as in shape and size. This study investigates sexual dimorphism in human face with the application of Automatic Homologous Multi-points Warping (AHMW) for 3D facial landmark by building a template mesh as a reference object which is thereby applied to each of the target mesh on Stirling/ESRC dataset containing 101 subjects (male $=47$, female $=54$ ). The semi-landmarks are subjected to sliding along tangents to the curves and surfaces until the bending energy between a template and a target form is minimal. Principal Component Analysis (PCA) is used for feature selection and the features are classified using Linear Discriminant Analysis (LDA) with an accuracy of $99.01 \%$.
\end{abstract}

Keyword: 3D sexual dimorphism; Automatic facial landmark; 3D geometric morphometrics; Multi-point warping; PCA; LDA 